Cahiers universitaires d'information sur l'Autriche

Finis Austriae : la chute de l'aigle bicéphale

\title{
100 Jahre Republik, 80 Jahre „Anschluss“ Bedenken zum Gedenkjahr 2018
}

Lucile Dreidemy

\section{(2) OpenEdition}

1 Journals

Édition électronique

URL : http://journals.openedition.org/austriaca/458

DOI : 10.4000/austriaca.458

ISSN : 2729-0603

Éditeur

Presses universitaires de Rouen et du Havre

\section{Édition imprimée}

Date de publication : 1 décembre 2018

Pagination : 257-263

ISBN : 979-10-240-1354-1

ISSN : 0396-4590

\section{Référence électronique}

Lucile Dreidemy, „100 Jahre Republik, 80 Jahre „Anschluss“ Bedenken zum Gedenkjahr 2018“,

Austriaca [Online], 87 | 2018, Online erschienen am: 01 März 2020, abgerufen am 28 Januar 2021. URL:

http://journals.openedition.org/austriaca/458 ; DOI: https://doi.org/10.4000/austriaca.458 


\section{Jahre Republik, 80 Jahre "Anschluss“" Bedenken zum Gedenkjahr 2018}

2018 war für Österreich ein mehrfaches Gedenkjahr. Im Rahmen von Konferenzen, Publikationen, Dokumentationen, Ausstellungen und Kunstperformances widmeten sich HistorikerInnen, PolitikerInnen und Kulturschaffende der Revolution von 1848, dem Ende des Ersten Weltkrieges, der Gründung der Ersten Republik 1918, dem „Anschluss“ 1938 und der Studentenrevolution von 1968. In Anlehnung an den thematischen Schwerpunkt dieser Austriaca-Ausgabe auf das Thema „Finis Austriae 1918“ empfiehlt sich ein besonderer Fokus auf den Stellenwert der Wendejahre 1918 und 1938 im Rahmen dieses Jubiläumsjahres, und insbesondere auf die Frage, was wie, bzw. nicht gedacht und bedacht wurde. Denn durch den Zoomeffekt auf spezifische historische Daten bringen Gedenkjahre bekanntlich immer die Gefahr mit sich, ein verzerrtes Geschichtsbild zu bieten, und somit auch Mythen zu nähren oder zu reaktualisieren.

\section{Mythen und Gedenken}

Wie hartnäckig Mythen im Diskurs über die Erste Republik und den "Anschluss" bleiben, bewies bereits der erste Höhepunkt des Gedenkjahres, nämlich das mediale Gedenken an die sogenannten „Schicksalstage" des März 1938, das von einem zum Teil sehr unkritischen Fokus auf die Person Kurt Schuschnigg begleitet wurde. So hörten etwa 1,1 Millionen Zuseher und Zuseherinnen am Sonntag, den 11. März 2018, die wichtigsten Ausschnitte aus Kurt Schuschniggs Abschiedsrede samt Schlussworte: „Gott schütze Österreich“" im ORF. Unterschiedliche Medien ließen auch Verwandte von Schuschnigg zu Wort kommen, in erster Linie seinen 91jährigen Sohn Kurt Schuschnigg Jr., den der Kurier-Journalist Georg Markus als „de[n] Kronzeuge[n] des Untergangs“ und „de[n] Sohn des letzten Kanzlers der Ersten Repub-

1. 1,1 Millionen lauschten Schuschniggs Rücktrittsrede im ORF, in Der Standard, 12.3.2018, URL: derstandard.at/2000075912549/Anschluss-im-ORF-1-1-Millionen-lauschten-derRuecktrittsrede-Kurt-Schuschniggs (letzter Zugriff: 10.2.2019). 
lik“" nannte, obwohl es die „Erste Republik“ anno 1938 schon seit fünf Jahren nicht mehr gab. Solche Verzerrungen und Verkürzungen blieben nicht auf den medialen Diskurs beschränkt.

\section{Mythen in der Geschichtswissenschaft}

Die Hartnäckigkeit von Mythen im wissenschaftlichen Diskurs über die Erste Republik, den Austrofaschismus und den „Anschluss“, zeigt sich exemplarisch an den zwei zentralen wissenschaftlichen Publikationen des Gedenkjahres 2018: Die gescheiterte Republik. Kultur und Politik in Österreich 1918-1938 des Politologen Anton Pelinka, und Die Erste Republik 1918-1938: Das Provisorium, des FPÖ-nahen Historikers Lothar Höbelt. So unterschiedlich der politische Background der Autoren auch sein mag, hier teilen sie nicht nur denselben Verlag und Untersuchungszeitraum, sondern auch einige fragwürdige Sichtweisen und Interpretationsmuster.

Beide Publikationen sind zunächst von einer ähnlichen Tendenz gekennzeichnet, das Ende der Ersten Republik als beinahe schicksalhaft zu präsentieren. Bei Pelinka geschieht es schon im Titel mit der Metapher des Scheiterns, die er auf den gesamten Staat bezieht. Wer ist diese Akteurin „Republik“, die laut Pelinka 1929 noch „,ihren Überlebenswillen“ (127) zeigte, und dann „abstürzte" (11)? Eine solche Subjektivierung ist nicht nur irreführend, sondern erweckt auch den Eindruck, die Republik wäre von selber zu Grunde gegangen, und nicht von klar benennbaren Akteuren, durch klar identifizierbare politische Entscheidungen und Maßnahmen aktiv zerstört worden. Die Wortwahl im Titel spiegelt den argumentativen roten Faden des ganzen Buchs wider, laut dem das Scheitern der Republik durch das Scheitern der politischen Kultur der Ersten Republik verursacht worden sei. „Denn die Kultur ignorierte weitgehend die Republik“, heißt es bereits auf Seite 1 - nochmals eine verstörende Subjektivierung ${ }^{3}$.

2. Kurt Schuschnigg jun.: „Was hätte mein Vater denn anderes tun sollen?', Interview mit dem Kurier-Journalisten und Sachbuchautoren Georg Markus, Kurier, 11.3.2018, URL: https:// kurier.at/politik/inland/kurt-schuschnigg-jun-was-haette-mein-vater-denn-anderes-tunsollen/313.492.505 (letzter Zugriff: 10.2.2019).

3. Zu Pelinkas unklarem Kulturbegriff, siehe die Klarstellungen des Germanisten Gerald Stieg in seiner detaillierten Buchrezension für die Plattform H-Soz-Kult: http://hsozkult. geschichte.hu-berlin.de/rezensionen/2017-4-043 (letzter Zugriff am 15.2.2019). 
Eine ähnliche Tendenz zur Personifizierung des Systems samt einhergehender Anonymisierung der Verantwortung findet sich auch bei Höbelt wieder: „Das politische System der Ersten Republik kippte früher oder später in ein autoritäres System. [...] Österreich folgte diesem Trend erst relativ spät, nicht durch einen klassischen Staatsstreich oder einen Bürgerkrieg, sondern ausgelöst durch eine Kette von Zufällen“.

Diesen rhetorischen Umwegen liegt die offenbare Scheu zugrunde, die politische Verantwortung des bürgerlichen Lagers am politischen Zerstörungsprozess der 1920er-1930er Jahre klar zu benennen. Diese Tendenz ist umso irritierender, als selbst ÖVP-nahe HistorikerInnen und ÖVP-PolitikerInnen im Laufe der letzten Jahrzehnte die Erkenntnisse der Forschung über den Austrofaschismus zunehmend akzeptiert und dementsprechend auch begonnen haben, das Ende der Republik als Ergebnis eines „Staatsstreichs" der Christlichsozialen zu betrachten ${ }^{4}$. Hingegen tendieren sowohl Höbelt als auch Pelinka dazu, die alte großkoalitionäre „Theorie der geteilten Schuld“ der Sozialdemokraten und Christlichsozialen am „Zusammenbruch“ der Ersten Republik zu reaktualisieren. Bei Pelinka drückt sich dieses großkoalitionäre Motiv bereits darin aus, dass er sein Buch den Gründungsvätern der Zweiten Republik widmet, namentlich Adolf Schärf und Karl Renner (zwei Sozialdemokraten, der letztere umstritten aufgrund seines „Ja“-zum "Anschluss" 1938) sowie den zwei ehemaligen Austrofaschisten und nachmaligen ÖVP-Bundeskanzlern Leopold Figl und Julius Raab.

Im Sinne einer vermeintlich geteilten Schuld heißt es bei Höbelt: „Die Suche nach einer Alternative zur parlamentarischen Demokratie hatte eine lange Tradition, aber der Weg in die Diktatur folgte einem unvorgesehenen und unvorhersehbaren Muster, war mehr von den Fehlern

4. Der ÖVP-nahe Historiker Helmut Wohnout spricht von „Rechts- und Verfassungsbruch“; vgl. Wohnout „Kontinuitäten und Brüche der österreichischen Parteiengeschichte am Beispiel der Christlichsozialen von den 1880er Jahren bis 1945“ in Georg Kastner / Ursula Mindler-Steiner / Helmut Wohnout (Hg.), Auf der Suche nach Identität: Festschrift für Dieter Anton Bindner, Wien: Lit 2015, S. 199-220, hier 207. 2003 sprach der damalige ÖVP-Nationalratspräsident Andreas Khol in einem Interview mit Dieter Kindermann von der Kronen Zeitung von einem „Staatsstreich“. Vgl. Khol, zitiert nach Oliver Rathkolb, Fiktion „Opfer" Österreich und die langen Schatten des Nationalsozialismus und der Dollfuß-Diktatur, Innsbruck, Wien, Bozen, StudienVerlag 2017, S. 95. Wenn es auf die Person Dollfuß ankommt, gewinnt aber oft der mythische Diskurs die Oberhand, so etwa als Khol anlässlich einer Podiumsdiskussion über mein Buch Der Dollfuß-Mythos: Eine Biographie des Posthumen (Wien, Böhlau, 2014) im Wien Museum im November 2014 wieder die alte Rhetorik vom „Märtyrer“ und „ersten Opfer Hitlers“ bemühte. 
der Gegner abhängig als von den eigenen Planungen “" So ähnlich heißt es auch bei Pelinka: „Es war das Fehlen einer starken demokratischen politischen Kultur, es war das Fehlen einer die Lagergrenzen überschreitenden demokratischen Zivilgesellschaft, das zum Ende der Republik führte“. Pelinka nuanciert zwar anschließend diese Ansicht, indem er erwähnt, dass „die drei staatsgründenden Lager der Ersten Republik [...] sich die Verantwortung für deren Scheitern [teilten] - aber nicht zu gleichen Teilen" “, was auf eine stärkere Verantwortung des bürgerlichen Lagers hindeuten mag. Doch im anschließenden Rückblick auf die Entscheidungsmomente der 1920er Jahre kommt der christlichsoziale Bundeskanzler Ignaz Seipel tendenziell besser weg als seine sozialdemokratischen Gegenspieler. So bewertet Pelinka Seipels „Koalitionsangebot“ an die Sozialdemokraten als „[e]inen letzten Versuch, eine auf Machtteilung aufbauende Konkordanz- und Konsensdemokratie zu etablieren“ (21). Die Ablehnung der Sozialdemokraten, wiederum, interpretiert er auf bedenkliche Weise teleologisch: „Angesichts der späteren Entwicklungen war die Ablehnung von Seipels Angebot letztlich die Zurückweisung einer wohl letzten Chance zur Rettung der Republik." (21) Pelinkas Argumentation hinkt vor allem deswegen, weil er von einem politischen Gleichgewicht der Kräfte ausgeht, das es 1931 längst nicht mehr gab: Bereits 1920 brachte das Ende der Großkoalition eine rasche Gegenoffensive der bürgerlichen Regierung mit sich. Der Abschluss der Genfer „Sanierungsanleihe“ 1922 gab dem bereits Bundeskanzler Seipel ein willkommenes Alibi für die Durchführung einer offensiven antisozialen und antidemokratischen Politik. Wie der Historiker Martin Kitchen bereits 1980 analysierte: "The Geneva Treaty was thus a further step towards the radicalisation of Austrian politics, and did much to increase the appeal of the radical right"'. Hinzu kam, dass die neue Kanzlerschaft Seipels ab 1926 eine Verschärfung des antimarxistischen Kurses der Regierung mit sich brachte. Dies zeichnete sich in erster Linie durch eine verstärkte Zusammenarbeit der Christlichsozialen mit der faschistischen Heimwehr aus, aber auch durch den Versuch eines Bündnisses mit Teilen des nationalsozialistischen Lagers im Vorfeld der Nationalratswahlen

5. Lothar Höbelt, Die Erste Republik 1918-1938: Das Provisorium, Wien, Böhlau, 2018, S. 364.

6. Anton Pelinka, Die gescheiterte Republik. Kultur und Politik in Österreich 1918-1938, Wien, Böhlau, 2018, S. 20.

7. Martin Kitchen, The Coming of Austrian Fascism, London, Croom Helm, 1980, S. 84f., zit. nach Florian Wenninger: „... werden mit aller Brutalität vorgehen.“ Zum Polarisierungsprozess der Zwischenkriegszeit in Österreich und seinen Nachwirkungen, $\mathrm{PhD}$, Universität Wien 2015, S. 58. 
$1927^{8}$. Im selben Jahr äußerte sich diese Radikalisierung im Freispruch der zwei Angehörigen der antimarxistischen Frontkämpfervereinigung, die anlässlich eines sozialdemokratischen Aufmarsches im burgenländischen Schattendorf im Jänner einen alten Mann und ein Kind getötet hatten. Dieses Urteil war der Auslöser für spontane Proteste am 15. Juli 1927, die von der Wiener Polizei blutig niedergeschlagen wurden, in die Geschichte eingegangen als Justizpalastbrand. Die Weltwirtschaftskrise 1929-30 verschärfte den antisozialen und antimarxistischen Kurs der bürgerlichen Regierung noch weiter. Wer diesen Hintergrund nicht berücksichtigt, kann die Ablehnung des „Koalitionsangebots“" 1931 durch die Sozialdemokraten nicht richtig einschätzen.

Auch bei der Deutung des Herrschaftssystems 1933-1938 werden die Erkenntnisse aus Jahrzehnten historiographischer Fortschritte einfach übergangen, und stattdessen alte, bereits oft sachlich dekonstruierte Mythen neuerlich bemüht. So postuliert etwa Pelinka ganz ungeachtet der einschlägigen Fachliteratur und ohne Quellenverweis, dass Dollfuß und Schuschnigg "nichts gegen die Demokratie an sich hatten“ (126) und dass Dollfuß die Zerstörung der Republik als unvermeidlichen Akt der Sicherung der österreichischen Unabhängigkeit gegenüber Hitlerdeutschland sehen wollte (143). Nach dieser Logik wird auch das „Versöhnungsabkommen“ mit Hitler-Deutschland im Juli 1936 als eine unvermeidliche Politik des Entgegenkommens gegenüber einem übermächtigen Gegner präsentiert (137).

Man könnte Pelinka zu Gute halten, dass der Schwerpunkt seiner Studie nicht auf dem Austrofaschismus, sondern auf der Ersten Republik liegt, die bekanntlich ab der diktatorischen Wende 1933 Säule für Säule zerstört, und mit der neuen „Maiverfassung" von 1934 endgültig begraben wurde. Trotz dieser unbestreitbaren Bruchlinie postulieren sowohl Höbelt als auch Pelinka bereits im Titel ihrer Werke, dass die Republik bis 1938 gedauert habe ${ }^{10}$. Pelinka rechtfertigt diese bedenk-

8. Vgl. Wenninger 2015, S. 55-60.

9. Es war außerdem kein Koalitionsangebot im engen Sinne, sondern eher die Einladung in eine Konzentrationsregierung unter Einschluss der Heimwehren, in der es eine überragende bürgerliche Mehrheit gegeben hätte. Danke an Florian Wenninger für diese Klarstellung.

10. Dieselbe Periodisierung findet sich auch im 2017 publizierten Sammelband des ÖVP-nahen Grazer Historikers Stefan Karner „Die umkämpfte Republik 1918-1938“, der als wissenschaftlicher Begleitband zur gleichnamigen Schwerpunktausstellung des „Hauses der Geschichte“ im Museum Niederösterreich herausgegeben wurde. Dort fand auch im Juli 2018 das Symposion „100 Jahre Republik. Geschichtsbilder einer Umbruchszeit (19181938)“ statt, mit einem Eröffnungsvortrag von Anton Pelinka. 
liche Periodisierung gleich zu Beginn seiner Einleitung: „Der Begriff 'Republik' bezieht sich nicht nur auf den Zeitraum zwischen der Gründung der Republik, 1918, und deren vorläufigem Ende, 1934. Um die Kontinuität über dieses Ende hinaus zu unterstreichen, ist auch der Zeitraum des autoritären, semifaschistischen, weder republikanischen noch demokratischen „Ständestaates" mit einbezogen." Von welcher Kontinuität kann man aber sprechen, wenn spätestens im Mai 1934 die republikanische Verfassung von 1929, deren Art. 1 lautete „Österreich ist eine demokratische Republik. Ihr Recht geht vom Volk aus", durch eine neue Verfassung ersetzt wurde, aus der ausgerechnet das Bekenntnis zur Demokratie und zur Republik sorgfältig gestrichen wurde, und dessen Art. 1 von nun an lautete: „Österreich ist ein Bundesstaat“.

Diese eindeutig politisch konservativ gefärbte Periodisierung mag in einem Titel vielleicht "runder" aussehen oder besser „reimen“, sie bleibt dennoch nicht nur historisch falsch, sondern auch irreführend, denn sie leugnet die Tatsache, dass die Republik nicht erst 1938 im Zug eines vermeintlichen „Anschlusses von außen“, sondern bereits fünf Jahre zuvor und von heimischen politischen Kräften zerstört wurde. Dadurch vertuscht sie auch, dass die Zerstörung des demokratischen, pluralistischen Rechtsstaates ab 1933 unter Dollfuß und die schrittweise Integration der „betont Nationalen“ unter Schuschnigg ab 1936 unmissverständlich den Weg zur Machtübernahme der Nazis bereiteten. Der problematische Charakter solcher unklaren Positionierungen wird anlässlich von Gedenkjahren wie dem letztjährigen besonders deutlich. Vor diesem Hintergrund darf man sich nicht wundern, wenn eine im März ausgestrahlte, neue ORF- Dokumentation zum „Anschluss“ mit dem Satz endete: „So endet der längste Tag, der letzte Tag der Ersten Republik. Sieben dunkle Jahre beginnen ${ }^{\text {"11 }}$.

Aus diesen Beobachtungen wird erschreckend deutlich, wie leicht Wissenschaftlichkeit auf dem Altar von Jubiläumsmarktopportunitäten geopfert werden kann. Die untersuchten Gedenkjahrprodukte haben gemeinsam, dass sie sich vor dem Hintergrund des Gedenkjahres damit begnügen, die Geschichte der Ersten Republik einzig aus der Perspektive des „Anschlusses“ zu deuten. Das Ergebnis ist eine eindeutig revisioni-

11. Siehe dazu exemplarisch die Doku Menschen \& Mächte: Der längste Tag - 18 Stunden, die Österreichs Schicksal entscheiden, Ein ORF-Film 2018, Realisation von Gerhard Jelinek, 51:30 Min, ausgestrahlt am 11.3.2018, ein Film wie er auch in den 1960ern hätte gedreht werden können. 
stische Tendenz, die die Erkenntnisse der Forschung über den Austrofaschismus vollkommen ignoriert, alte mythische Topoi (insbesondere die These der geteilten Schuld) reaktiviert, und die Rolle des DollfußSchuschnigg-Regimes als Wegbereiter des Nationalsozialismus relativiert.

Lucile DreIDEMY

\section{Une authentique rencontre : Martin Buber de Dominique Bourel}

Dominique Bourel a mis en exergue à sa biographie de Martin Buber cette belle citation de ce dernier : "Jedes wirkliche Leben ist Begegnung. » C'est, en une phrase, la quintessence de ce travail qu'on ne saurait résumer ni en quelques lignes, ni même en quelques pages : il s'agit de rendre hommage à une monumentale biographie publiée en $2015^{12}$ dont a paru fin 2017 la traduction allemande ${ }^{13}$ - occasion pour nous de relire la version originale et de comparer les deux pour tenter, ici, d'en dégager les grandes lignes.

À côté de et après Mendelssohn $n^{14}$, Buber représente vingt années de recherches et de rédaction, d'innombrables textes et documents à étudier, une infinité de lettres, 50000 dit Bourel, à prendre en considération. Résultat : 700 pages de texte et 300 de notes et de bibliographie pour la version allemande. Un long voyage donc, touffu et dense, où le lecteur assiste par le biais du penseur juif, à la fin du XIX $^{e}$ et à deux tiers du $\mathrm{xx}^{\mathrm{e}}$ siècle, donc à deux guerres mondiales, la chute de deux empires (des Habsbourg et des Hohenzollern), à l'avènement et à la chute de la première république allemande, à la persécution des juifs et à la Shoah dans les territoires sous domination nazie et finalement à la création de l'État d'Israël en Palestine.

12. Dominique Bourel, Martin Buber, sentinelle de l'humanité, Paris, Albin Michel, 2015.

13. Dominique Bourel, Martin Buber. Was es heißt ein Mensch zu sein, Horst Brühmann (trad.), Gütersloh, Gütersloher Verlagshaus, 2017.

14. Dominique Bourel, Moses Mendelssohn. La naissance du judaïsme moderne, Paris, Gallimard, 2004. 